Supporting Information

\title{
Anion-Assisted Delivery of Multivalent Cations to Inert Electrodes
}

\author{
Artem Baskin $^{*[a]}$, John W. Lawson ${ }^{[a]}$, David Prendergast $t^{[b][c]}$
}

${ }^{a}$ NASA Ames Research Center, Moffett Field, California 94035, USA

${ }^{\mathrm{b}}$ Joint Center for Energy Storage Research, Lawrence Berkeley National Laboratory, Berkeley, CA 94720, USA

${ }^{\mathrm{c}}$ The Molecular Foundry, Lawrence Berkeley National Laboratory, Berkeley, CA 94720, USA

Correspondence to: artem.i.baskin@nasa.gov 


\section{Table of Contents}

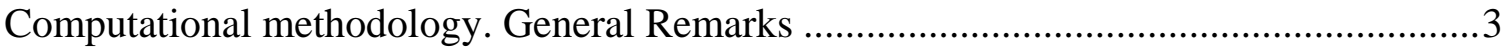

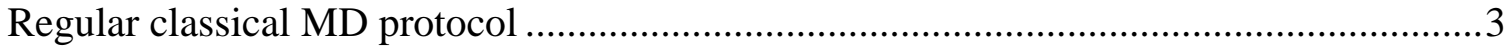

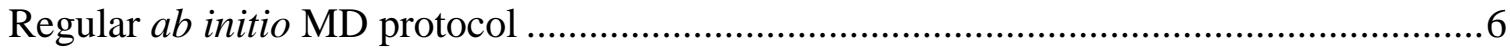

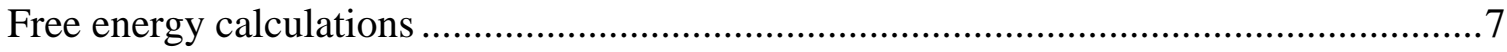

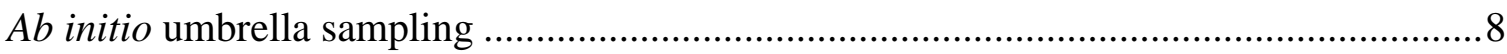

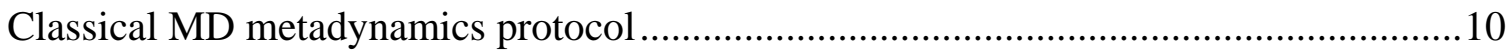

Polarization effect on the graphene induced by the $\mathrm{Mg}$-cation in THF ..........................12

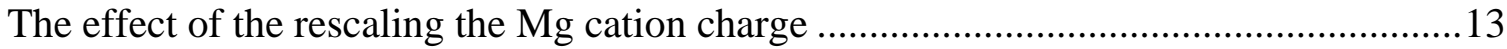

Solvation structure dynamics of the $\mathrm{MgTFSI}^{+}$ion pair in THF next to the negatively charged

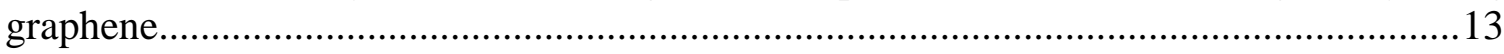

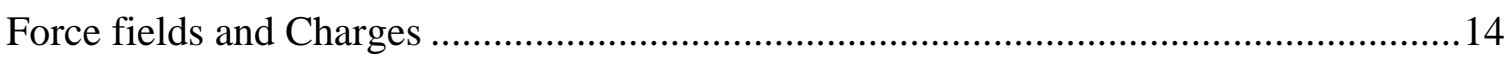

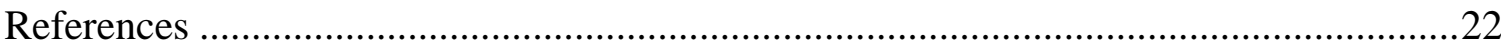


We carry out regular classical and ab initio molecular dynamics simulations (MD) of the $\mathrm{Mg}$ cation, its ion pairs and various aggregates with the bis-trifluorosulfonimide anion (TFSI') solvated in tetrahydrofuran (THF) in the dilute limit and at finite concentration of $\mathrm{MgTFSI}_{2}$ salt near a single graphene sheet (neutral or negatively charged) in a half-cell setup (one graphene sheet per a unit cell). Under periodic boundary conditions, these supercells may demonstrate symmetric or asymmetric distributions of ionic species in the liquid electrolyte as a function of perpendicular distance (z-direction) from the graphene sheet. In the asymmetric case, the majority of ionic species are located near one side of the graphene electrode and almost none on the other side. This is specifically true for simulations with negatively charged graphene where the charge balancing provided by species in the electrolyte occurs only on one side of this quasi-twodimensional electrode - i.e., breaking inversion symmetry. However, our focus here is not on studying the structure of the double layer. Instead, we focus on ion solvation structure and its modifications due to proximity to the interface.

We use equilibrated structures as input for the metadynamics protocol [1,2] and umbrella sampling [3,4] to evaluate free energy profiles along the collective variables of interest. Throughout all classical MD calculations (regular and metadynamics protocols) the nonpolarizable Generalized Amber force field [5,6] (GAFF) was used for ions and solvent molecules. The charge of the Mg cation was set +2 unless stated otherwise (see details below for modeling $\mathrm{MgTFSI}^{+}$ion pairs and neutral aggregates). For the graphene sheet, we used the force field optimized previously for graphene-water interactions [7]. General neutrality of the simulation cells was maintained by balancing the total number of cations and anions in the supercell with the charge on the graphene electrode (if any) or using a neutralizing background if a single ionic species per supercell was modeled. The parameters of the force field for the Mg-cation, TFSIanion, THF solvent and graphene, along with other computational details are listed below. Throughout the $a b$ initio modeling (MD and umbrella sampling), we used a simulation cell that contained a single graphene sheet adjacent to an equilibrated volume of THF molecules with a single $\mathrm{Mg}$ cation. See details below.

\section{Regular classical MD protocol}

Classical molecular dynamics (MD) simulations were performed using the LAMMPS simulation package. [8] Long-range electrostatic interactions were treated within the particlemesh Ewald (PME) method with a cutoff distance $1.0 \mathrm{~nm}$ with grid spacing in $k$-space of $10^{-5}$. A cut-off of $1.0 \mathrm{~nm}$ with a spline from 0.9 to $1.0 \mathrm{~nm}$ was used for Lennard-Jones interactions. The relaxation of the initial structures was performed in two steps, first using steepest descent with a convergence criterion of $10^{-4} \mathrm{kcal} \mathrm{mol}^{-1}$ for energies and $10^{-4} \mathrm{kcal} \mathrm{mol}^{-1} \AA^{-1}$ for forces. The systems were first heated to the target temperature in the canonical ensemble (NVT). To remove any "memory" effects, the systems were first melted at $500 \mathrm{~K}$ and then annealed back to the target temperature (e.g., $298 \mathrm{~K}$ ) three times (evolving the trajectory $2 \mathrm{~ns}$ for annealing each step). Then, isothermal-isobaric (NPT, isotropic $P=1 \mathrm{~atm}$, at fixed target temperature) simulations were performed for $2 \mathrm{~ns}$ ( 2 fs time step) to obtain the correct density using a Nose-Hoover thermostat [9] and Nose-Hoover barostat. [10] The temperature coupling constant was $0.1 \mathrm{ps}$, the pressure piston constant was 2.0 ps. Afterwards, NVT simulations were performed at target temperature for $1 \mathrm{~ns}$ ( $1 \mathrm{fs}$ time step) to equilibrate. Structural properties were obtained from $5 \mathrm{~ns}$ MD 
simulation runs with an integration time step 1 fs in the NVT ensemble with coordinates and velocities saved every 5 ps for post-trajectory analysis.

We performed simulations of the Mg cation, TFSI anion, and the ion pair $\mathrm{MgTFSI}^{+}$in the dilute limit (one species per box) and at finite MgTFSI2 salt concentration in THF $(0.12 \mathrm{M}-0.25$ M) next to a single sheet of graphene. See Figure S1 for a typical simulation cell. The graphene sheet was unfixed during the equilibration and thermalization but it was fixed (we zero the forces acting on graphene C-atoms) during the NPT and the production NVT cycles (see Table S1 for parameters of simulations). For each case, when a single ionic species was placed in a box of solvent molecules, equilibrated as described above, a neutralizing background was used (when necessary) to properly account for electrostatic interactions. The negative charge, when applied, to the graphene was evenly distributed over the all carbon atoms. For the finite concentration simulations, the specific number of ionic species was then used to balance out the negative charge on the graphene. All the simulations were performed at $\mathrm{T}=298 \mathrm{~K}$. The relaxation of the supercell dimensions during NPT simulations were not considered to depend significantly on the specific distribution of ionic species in the electrolyte. As validation, for these large simulation cells, we do not see a noticeable difference in computed pressures during NVT simulations depending on the location of the species.

Figure S1. A typical simulation unit cell (half-cell setup, for parameters of the equilibrated boxes see Table S1). A species of interest near the graphene is highlighted.

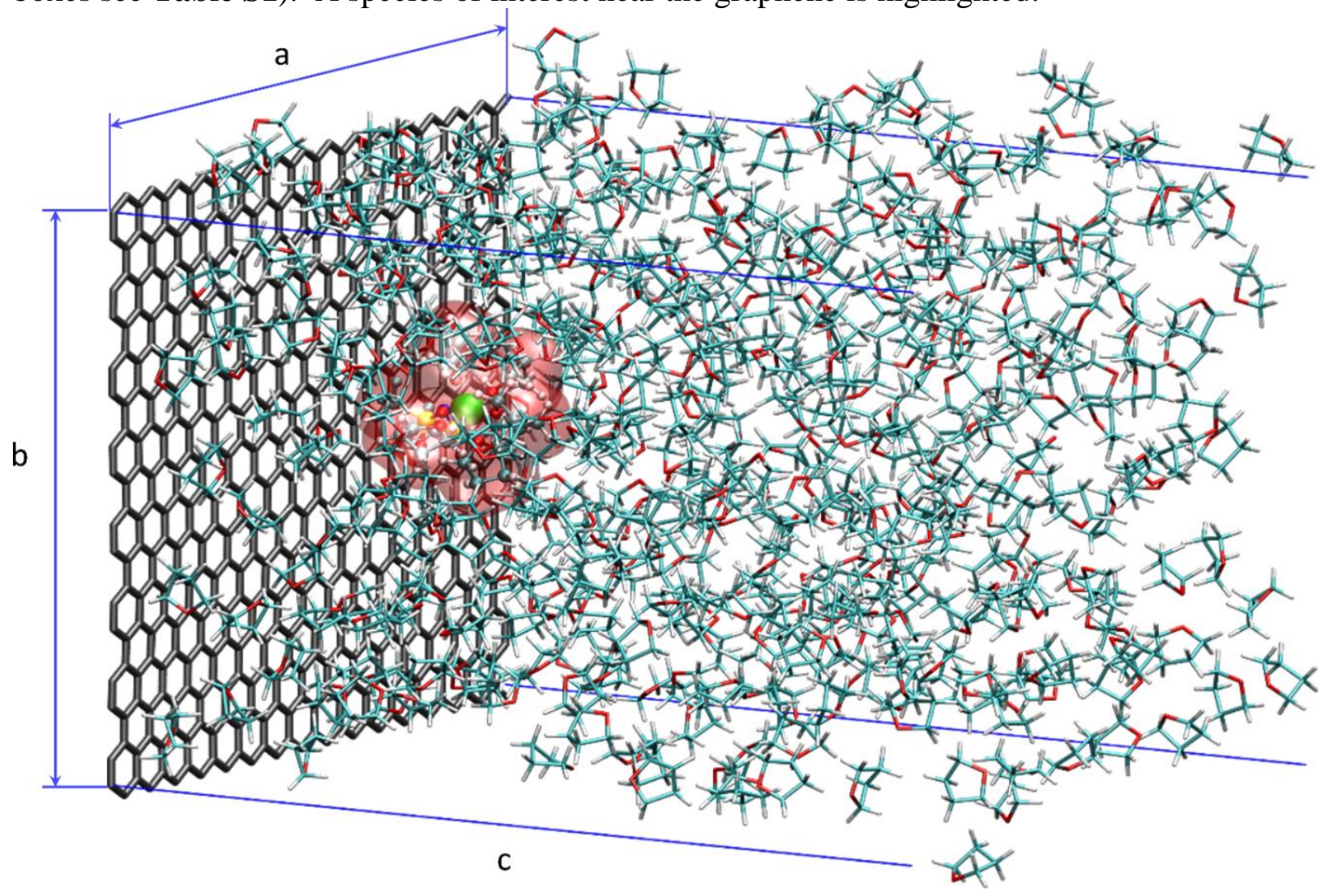


Table S1. Parameters of classical MD simulations. 1D or 2D indicates the use for 1D or 2D free energy metadynamics sampling

\begin{tabular}{|c|c|c|c|}
\hline Simulations & \# of molecules in the box & $\begin{array}{l}\text { Equilibrated } \\
\text { box parameters }(\AA)\end{array}$ & Additional details \\
\hline $\begin{array}{l}\text { THF box next to the } \\
\text { neutral graphene }\end{array}$ & $\begin{array}{c}512 \mathrm{C} \text { atoms (graphene), } \\
\text { 378THF }\end{array}$ & $39.348 \times 34.131 \times 48.985$ & \\
\hline $\begin{array}{l}\text { Mg cation/THF next } \\
\text { to the neutral } \\
\text { graphene }(1 \mathrm{D}, 2 \mathrm{D})\end{array}$ & $\begin{array}{l}512 \mathrm{C} \text { atoms (graphene), } \\
1 \mathrm{Mg} \text { cation, } 378 \mathrm{THF}\end{array}$ & $39.225 \times 34.025 \times 48.832$ & $\mathrm{Q}(\mathrm{Mg})=+2$ \\
\hline $\begin{array}{l}\mathrm{Mg} \text { cation/THF, next } \\
\text { to the neutral } \\
\text { graphene ( } 2 \mathrm{D})\end{array}$ & $\begin{array}{l}512 \mathrm{C} \text { atoms (graphene), } \\
1 \mathrm{Mg} \text { cation, } 378 \mathrm{THF}\end{array}$ & $39.227 \times 34.027 \times 48.835$ & $\mathrm{Q}(\mathrm{Mg})=+1.6$ \\
\hline $\begin{array}{l}\text { Mg cation/THF next } \\
\text { to the charged } \\
\text { graphene }(2 \mathrm{D})\end{array}$ & $\begin{array}{l}512 \mathrm{C} \text { atoms (graphene), } \\
1 \mathrm{Mg} \text { cation, } 378 \mathrm{THF}\end{array}$ & $39.326 \times 34.113 \times 48.958$ & $\begin{array}{c}\mathrm{Q}(\mathrm{Mg})=+2 \\
\mathrm{Q}(\text { graphene })=-2\end{array}$ \\
\hline $\begin{array}{l}\text { Mg cation/THF next } \\
\text { to the charged } \\
\text { graphene (1D) }\end{array}$ & $\begin{array}{l}512 \mathrm{C} \text { atoms (graphene), } \\
1 \mathrm{Mg} \text { cation, } 378 \mathrm{THF}\end{array}$ & $39.298 \times 34.088 \times 48.923$ & $\begin{array}{c}\mathrm{Q}(\mathrm{Mg})=+2 \\
\mathrm{Q}(\text { graphene })=-10\end{array}$ \\
\hline $\begin{array}{l}\mathrm{MgTFSI}^{+} / \mathrm{THF}, \text { next } \\
\text { to the neutral } \\
\text { graphene(1D, 2D) }\end{array}$ & $\begin{array}{l}512 \mathrm{C} \text { atoms (graphene), } \\
1 \mathrm{MgTFSI}^{+}, 377 \mathrm{THF}\end{array}$ & $39.235 \times 34.033 \times 48.844$ & $\begin{array}{l}\mathrm{Q}(\mathrm{Mg})=+1.8 \\
\mathrm{Q}(\mathrm{TFSI})=-0.8\end{array}$ \\
\hline $\begin{array}{l}\mathrm{MgTFSI}^{+} / \mathrm{THF}, \text { next } \\
\text { to the charged } \\
\text { graphene }(1 \mathrm{D}, 2 \mathrm{D})\end{array}$ & $\begin{array}{l}512 \mathrm{C} \text { atoms (graphene), } \\
1 \mathrm{MgTFSI}^{+}, 377 \mathrm{THF}\end{array}$ & $39.234 \times 34.033 \times 48.844$ & $\begin{array}{c}\mathrm{Q}(\mathrm{Mg})=+1.8 \\
\mathrm{Q}(\mathrm{TFSI})=-0.8 \\
\mathrm{Q} \text { (graphene) }=-10\end{array}$ \\
\hline $\begin{array}{l}\text { TFSI } / \text { THF, next to } \\
\text { the neutral graphene } \\
\text { (1D) }\end{array}$ & $\begin{array}{l}512 \mathrm{C} \text { atoms (graphene), } \\
1 \text { TFSI anion, } 377 \mathrm{THF}\end{array}$ & $39.234 \times 34.033 \times 48.843$ & $\mathrm{Q}(\mathrm{TFSI})=-0.8$ \\
\hline $\begin{array}{l}\mathrm{MgTFSI}_{2} / \mathrm{THF}, \text { next } \\
\text { to the neutral } \\
\text { graphene (2D) }\end{array}$ & $\begin{array}{l}512 \mathrm{C} \text { atoms (graphene), } \\
5 \mathrm{Mg} \text { cations, } 10 \mathrm{TFSI} \\
\text { anions, } 364 \mathrm{THF}\end{array}$ & $39.362 \times 34.144 \times 49.002$ & $\begin{array}{l}\mathrm{Q}(\mathrm{Mg})=+1.6 \\
\mathrm{Q}(\mathrm{TFSI})=-0.8\end{array}$ \\
\hline $\begin{array}{l}\mathrm{MgTFSI}_{2} / \mathrm{THF}, \text { next } \\
\text { to the charged } \\
\text { graphene (2D) }\end{array}$ & $\begin{array}{c}512 \mathrm{C} \text { atoms (graphene), } \\
10 \mathrm{Mg} \text { cations, } 10 \mathrm{TFSI} \\
\text { anions, } 359 \mathrm{THF}\end{array}$ & $39.321 \times 34.108 \times 48.951$ & $\begin{array}{c}\mathrm{Q}(\mathrm{Mg})=+1.6 \\
\mathrm{Q}(\mathrm{TFSI})=-0.8 \\
\mathrm{Q}(\text { graphene })=-8\end{array}$ \\
\hline
\end{tabular}

As the charge for the force filed parameters for the TFSI anion are optimized for its overall charge equal to -0.8 , the charge of the $\mathrm{Mg}$ cation is adjusted accordingly to the composition of the system and is indicated in the Table S1 for each simulation. The effective area, S, of the graphene electrode (512 C atoms) at rest is $1208.35 \AA^{2}$, so the effective surface charge density, in units of $\mu \mathrm{C} / \mathrm{cm}^{2}$ is calculated accordingly: $\frac{\# \text { of electrons }}{S\left[\AA^{2}\right]} \times 1602$. 


\section{Regular ab initio MD protocol}

We used ab initio MD (AIMD) simulations to explore solvation structures of the Mg cation in THF at various distances from the graphene and associated costs as the cation approaches the electrode. Thermalized equilibrium structures for fixed distances ( $3 \AA$, $4 \AA$, $5 \AA$, $6.5 \AA$ and 8.76 $\AA$ ) of the Mg cation from the fixed plane of the graphene sheet were obtained. The unit cells were identical for all $a b$ initio simulations: the graphene sheet (112 C atoms), $1 \mathrm{Mg}$ cation and $25 \mathrm{THF}$ molecules, box size 17.248x17.071092x17.1 $\AA^{3}$ (see Figure S2). The total charge of the unit cell was set to +2 . Using a sampling time step of $0.5 \mathrm{fs}$, AIMD trajectories of ca. $5 \mathrm{ps}$ within the canonical NVT ensemble at $300 \mathrm{~K}$ with the Nose-Hoover thermostat [11] (with characteristic timestep of $100 \mathrm{fs}$, and a Nose-Hoover chain of length 3) and periodic boundary conditions were carried out using the Quickstep module of the CP2K package [12]. For non-neutral systems, the universal background compensating charge scheme and the Poisson periodic three-dimensional solver were used, and the total energy was sampled at the $\Gamma$-point of the first Brillouin zone. The valence electrons were treated explicitly at the DFT level using the revPBE parametrization of the exchange-correlation functional, a triple- $\zeta$ basis set with two additional sets of polarization functions (TZV2PX) [13], and a 320 Ry energy grid cutoff. The core electrons on all atoms were treated using norm-conserving Goedecker-Teter-Hutter (GTH) pseudopotentials. [14] Longrange dispersion forces were treated with the DFTD3 empirical Grimme correction. [15].

An additional note on the size of the $a b$ initio unit cells. Due to the computational cost and the lack of ionic screening we do not use a counter ion(-s) in the unit cell which implies possible strong charge-charge interactions between the periodic images. However, here we are not interested in the total energy (referenced to a common value). Moreover, the cation coordination is a local phenomenon. Therefore, the dipole-dipole coupling between image cells should not play a noticeable role in defining a local cation environment as long as the size of the unit cell precludes the overlap between the cation solvation spheres. The latter is what defines the minimum lateral size of the unit cell. The chosen dimensions of the unit cell satisfy this requirement.

Figure S2. A typical $a b$ initio simulation unit cell (half-cell setup). The Mg coordination with THF molecules is highlighted.

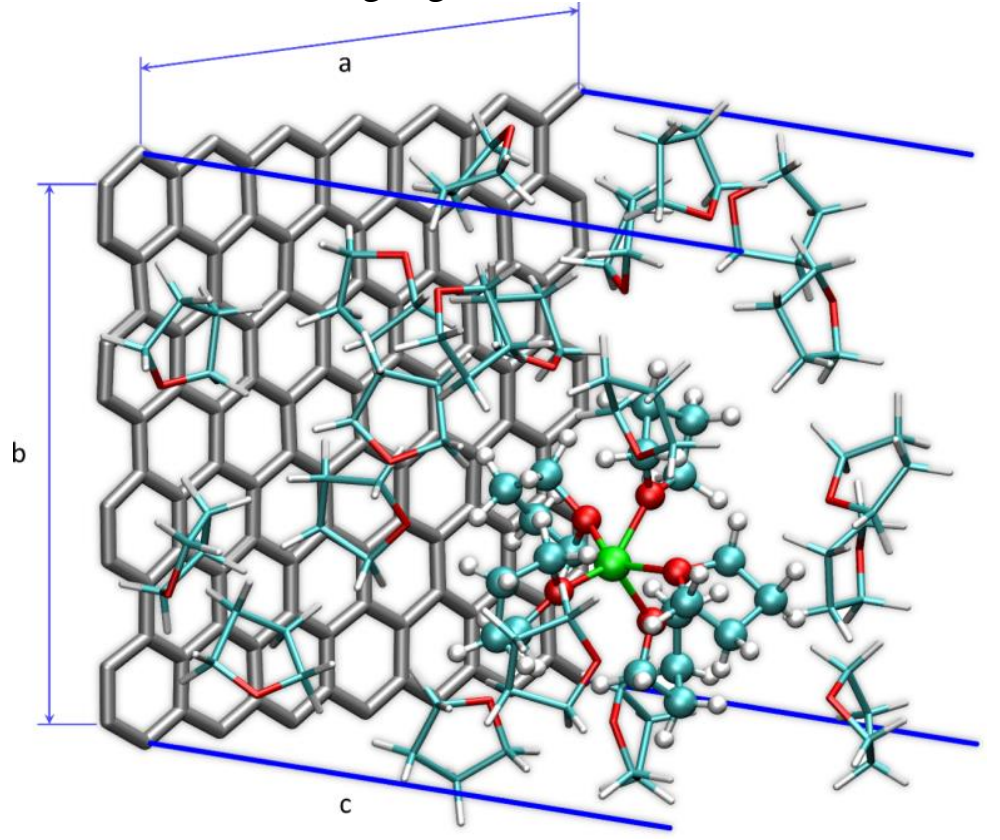




\section{Free energy calculations}

We performed $a b$ initio and classical free energy sampling to evaluate the free energy profiles along specific collective variables (CVs): (1) the ion-solvent coordination number (denoted $\mathrm{CN}$ in figures) (effectively, the number of oxygen atoms of THF molecules within the first coordination sphere of the $\mathrm{Mg}$ cation); (2) the ion-anion coordination number (the number of oxygen atoms of TFSI anions about Mg) - see further details below; (3) the distance (denoted d in figures) between the $\mathrm{Mg}$ cation and the graphene plane; and (4) the ion-anion molecule distance (between the $\mathrm{Mg}$ cation and the center of mass of the S-N-S portion of the TFSI anion). We used these CV's and their combinations to explore 1D profiles and 2D free energy surfaces.

Specifically, we use the umbrella sampling technique [3,4] and the metadynamics [1,2] protocol to evaluate the potential of mean force along the collective variables. For a system of $\mathrm{N}$ particles in a canonical ensemble at temperature $\mathrm{T}$, the probability density associated with a generalized coordinate $\lambda$ (our preferred $\mathrm{CV}$ ) having a value of $\lambda_{0}$ can be expressed in terms of a generalized partition function:

$$
\Omega\left(\lambda_{0}\right)=\int d q^{3 N} d p^{3 N} \delta\left(\lambda-\lambda_{0}\right) e^{-\beta H\left(q^{N}, p^{N}\right)},
$$

where $q$ and $p$ are conjugated generalized coordinates and momenta of particles and we assume that additional constraints associated with general integrals of motion are already subtracted. Accordingly, the configurational part of the partition function can be written as

$$
Z\left(\lambda_{0}\right)=\int d q^{3 N} \delta\left(\lambda-\lambda_{0}\right) e^{-\beta H\left(q^{N} ; \lambda\right)} .
$$

In a basis with no cross terms involved, we rewrite the probability density as:

$$
\Omega(\lambda)=\int d q^{3 N-1} d p^{3 N-1} d P_{\lambda} e^{-\beta H\left(q^{N}, p^{N}, P_{\lambda}\right)},
$$

where we integrate out all degrees of freedom and conjugated momenta except the coordinate of interest, $\lambda$. The potential of mean force (PMF) is then defined as $W(\lambda)=-k T \ln \Omega(\lambda)+$ const. The integration constant is chosen such that $\min \{W(\lambda)\}=W\left(\lambda_{\min }\right)=0$, where $\lambda_{\text {min }}-$ is the value of the $\mathrm{CV}$ at which $W(\lambda)$ reaches its global minimum.

For a given collective variable, $\lambda$, the PMF $W(\lambda)$ can also be expressed as follows:

$$
W(\lambda)=k T \ln \left[\int e^{+\beta H\left(q^{N-1} ; \lambda\right)} \operatorname{Pr}\left(q^{N-1} ; \lambda\right) d q^{N-1}\right]
$$

where $H\left(q^{N} ; \lambda\right)$ is the energy of a conditional configuration $\left(q^{N-1} ; \lambda\right)$ and

$$
\operatorname{Pr}\left(q^{N-1} ; \lambda\right)=\frac{e^{-\beta H\left(q^{N-1} ; \lambda\right)}}{\int e^{-\beta H\left(q^{N-1} ; \lambda\right)} d q^{N-1}}
$$

is the probability of that conditional configuration directly related to the partition function $Z(\lambda)$. The sampling effectively integrates out all the other degrees of freedom, except $\lambda$, and in this light it can be viewed as thermodynamic integration over the free energy basin (defined by the chosen inverse temperature, $\beta$ ), because the associated values of the PMF at various values of the reaction coordinate $\lambda$ account for the Boltzmann statistics of states $\left(q^{N-1} ; \lambda\right)$.

\section{Definition of the coordination number}

We use the following functional form of the coordination number $\mathrm{CN}$ :

$$
C N=\sum_{i=1}^{N} \frac{1-\left(\frac{r_{i}}{r_{o}}\right)^{p}}{1-\left(\frac{r_{i}}{r_{O}}\right)^{q}} \text {, where } \mathrm{p}=6, \mathrm{q}=12, \mathrm{r}_{\mathrm{i}}-\text { is the distance between the ion and the } \mathrm{i} \text {-th }
$$

coordinating atom (e.g., oxygen atom in THF or TFSI), $r_{0}$ - the cut-off radius that separates atoms that belong to the solvation sphere from those that do not, and i runs over the range that includes all possible coordinating atoms (e.g., all oxygen atoms in the simulation box). Cut-off radii were 
defined using the following procedure: For a regular classical or AIMD protocol, we define the $\mathrm{CN}$ of the ion by calculating the integrated $\mathrm{g}(\mathrm{r})$. We then vary the cut-off radius $\mathrm{r}_{\mathrm{o}}$ to achieve the same $\mathrm{CN}$, using the functional form above, for unbiased classical or $a b$ initio MD, as defined by the regular (AI)MD protocol. Within the context of a free energy sampling simulation, this would imply that no Gaussians have been added yet to define a bias potential within metadynamics, or that the force constant within umbrella sampling has been set to zero, $\mathcal{H}=0$.

Strictly speaking, the values of $\mathrm{CN}$ at which the free energy profile has a minimum depends on the parameters of the function chosen to calculate $\mathrm{CN}\left(\mathrm{r}_{\mathrm{o}}, \mathrm{p}\right.$ and $\left.\mathrm{q}\right)$. The same holds true for the kinetic barriers that separate the minima (if any). However, once $r_{o}$ is found (for given $p$ and q), the minima and the kinetic barriers of the free energy are well defined. Assuming that at least one free energy minima is well separated from other stable states (if any), then the described above procedure of defining $r_{o}$ (for a given $p$ and $q$ ) based on the running integrated value of $g(r)$ gives physically motivated values of parameters of the $\mathrm{CN}$ function. The change of exponents $\mathrm{p}$ and q will likely result in the change of the cut-off radius $r_{0}$ in order to get the coordination number (-s) similar to that from $\mathrm{g}(\mathrm{r})$. Given the fact that $\mathrm{g}(\mathrm{r})$ is an experimentally measurable quantity (so is the coordination number), $\mathrm{p}, \mathrm{q}$ and cut-off radius are connected and $\mathrm{p}$ and $\mathrm{q}$ values may change only in a certain range, so does $r_{0}$. Therefore, for physically meaningful values of $\mathrm{p}$, $\mathrm{q}$ and cut-off radius, the kinetic barriers between different stable coordination states should not change significantly. An additional study is needed to get quantitative correlations.

\section{Ab initio umbrella sampling}

For each distance (both the coordinates of graphene and the $\mathrm{Mg}$ cation were fixed), we defined the sampling windows for $\mathrm{CN}$ (coordination number of the $\mathrm{Mg}$ cation with respect to oxygen atoms of THF molecules) and apply harmonic umbrella potentials of the form $U_{i}(C N)=$ $\varkappa_{i}\left(C N-C N_{i}\right)^{2}$, where $\mathrm{i}$ refers to the $\mathrm{i}$-th window, $\varkappa_{i}$ is the force constant for the $\mathrm{i}$-th window and $C N_{i}$ is the target coordination number for the $\mathrm{i}$-th window. For each umbrella window, a trajectory of 20 ps was collected after 2 ps of pre-equilibration. To guarantee the stability of the umbrella sampling trajectories, as excessively high $\mathcal{U}$ values can cause chemical decomposition of the THF molecules: if an initial configuration is characterized by $\mathrm{CN}$ that is far from the target $\mathrm{CN}$ value, the initial configurations for each distance $\mathrm{d}$ were pre-optimized by first applying soft force constants $\varkappa_{i} \approx 0.05-0.1 a$. u. to bring the structures closer to the required target values of $\mathrm{CN}$. The width of $\mathrm{CN}$ windows is 0.1 . Overall simulation time of these five systems is $3.080 \mathrm{~ns}$. The weighted histogram analysis method (WHAM) [16] was used to construct the free energy profiles from these histograms. The parameters $\left(\mathrm{r}_{0}, \varkappa_{i}\right)$ for each simulation are summarized in Table S2.

Table S2. Parameters of ab initio umbrella samplings.

\begin{tabular}{|c|c|c|c|c|c|}
\hline $\mathrm{Mg}^{2+} / \mathrm{THF}$ & $C N_{i}$ & 2.5 & 2.6 & $\ldots$ & 4.5 \\
\hline $\begin{array}{c}\mathbf{a}=3.0 \mathrm{~A} \\
r_{o}=3.28 \AA \\
21 \text { windows }\end{array}$ & $\begin{array}{l}\mathcal{H}_{i}, \\
\text { a.u. }\end{array}$ & \multicolumn{4}{|c|}{0.5} \\
\hline $\mathrm{Mg}^{2+} / \mathrm{THF}$ & $C N_{i}$ & 3.0 & 3.1 & & 5.0 \\
\hline $\begin{array}{c}\mathbf{d}=4.0 \mathrm{~A} \\
r_{o}=3.28 \AA \\
21 \text { windows }\end{array}$ & $\begin{array}{l}\mathcal{H}_{i}, \\
\text { a.u. }\end{array}$ & \multicolumn{4}{|c|}{0.5} \\
\hline $\mathrm{Mg}^{2+} / \mathrm{THF}$ & $C N_{i}$ & 3.0 & 3.1 & $\ldots$ & 5.5 \\
\hline
\end{tabular}




\begin{tabular}{|c|c|c|c|c|c|}
\hline $\begin{array}{c}\mathbf{d}=\mathbf{5 . 0} \AA \\
r_{o}=3.28 \AA \\
26 \text { windows }\end{array}$ & $\begin{array}{l}\mathcal{H}_{i}, \\
\text { a.u. }\end{array}$ & \multicolumn{4}{|c|}{0.5} \\
\hline $\mathrm{Mg}^{2+} / \mathrm{THF}$ & $C N_{i}$ & 3.0 & 3.1 &. & 6.5 \\
\hline $\begin{array}{c}\mathbf{d}=\mathbf{6 . 5} \mathrm{A} \\
r_{o}=3.28 \AA \\
36 \text { windows }\end{array}$ & $\begin{array}{l}\mathcal{H}_{i}, \\
\text { a.u. }\end{array}$ & \multicolumn{4}{|c|}{0.5} \\
\hline $\mathrm{Mg}^{2+} / \mathrm{THF}$ & $C N_{i}$ & 3.0 & 3.1 & .. & 6.5 \\
\hline $\begin{array}{c}\mathbf{d}=\mathbf{8 . 7 6} \AA \\
r_{o}=3.28 \AA \\
36 \text { windows }\end{array}$ & $\begin{array}{l}\mathcal{H}_{i}, \\
\text { a.u. }\end{array}$ & \multicolumn{4}{|c|}{0.5} \\
\hline
\end{tabular}

Accuracy of the free energy calculations from umbrella sampling critically depends on the density of the sampling windows and the overlapping of adjacent probability distributions. Here we used parameters that were proven [16] to provide an efficient overlapping and thus guarantee the convergence of the WHAM algorithm.

Given the computational costs of ab initio MD, here we analyze the solvation structure of an isolated $\mathrm{Mg}$ cation at fixed distances from the neutral graphene electrode and use the distance dependence of the ensemble average total energy $\langle\operatorname{Etot}(\mathrm{d})\rangle$ at solvation configurations (coordination number $\mathrm{CN}$ ) that correspond to the minima of the free energy $\Delta \mathrm{G}(\mathrm{CN}) \rightarrow$ min. As one expects, the major costs of the cation approaching to the electrode are related to the deformation of its solvation sphere. Therefore, we can use $\langle\operatorname{Etot}(d)\rangle_{\{\min (\mathrm{CN})\}}$ as a proxy to a slice along the optimal pathway $\mathrm{CN}=\mathrm{CN}_{\min }(\mathrm{d})$ on the true $2 \mathrm{D}$ free energy surface $\Delta \mathrm{G}(\mathrm{CN}, \mathrm{d})$.

For every global minimum with respect to $\mathrm{CN}$ of the $\mathrm{Mg}$ cation solvation free energy for a given fixed distance (indicated by arrows in Figure 3, main text) we calculated averaged internal energy $\left\langle E\left(d_{i}\right)\right\rangle_{t}$, where $d_{i}$ is the fixed distance $(3 \AA, 4 \AA, 5 \AA, 6.5 \AA$ and $8.76 \AA$ ), using the constrained trajectories $(\approx 10 \mathrm{ps})$. Since the bias $U(C N) \approx 0$ at $C N=C N_{\text {target }}$ the internal energy $E$ has no additional contribution from the biased umbrella sampling potential. In Table S3 we show the raw averaged energies output from $\mathrm{cp} 2 \mathrm{k}$ and the standard quadratic deviation. The profile of $\left\langle E\left(d_{i}\right)\right\rangle_{t}$ referenced to $\langle E(d=8.7 \AA)\rangle_{t}$ is shown in Fig. S3.

Table S3. Averaged energies and standard deviations for each distance and optimal coordination number.

\begin{tabular}{|c|c|c|c|c|c|}
\hline Distance, $\AA$ & $8.7(\mathrm{CN}=5.0)$ & $6.5(\mathrm{CN}=5.0)$ & $5.0(\mathrm{CN}=4.0)$ & $4.0(\mathrm{CN}=4.0)$ & $3.0(\mathrm{CN}=3.0)$ \\
\hline$\left\langle E\left(d_{i}\right)\right\rangle_{t}$, a.u. & -1790.014128 & -1789.9691595 & -1789.9483883 & -1789.9475 & -1789.9347591 \\
\hline$\sqrt{\sigma^{2}}$, a.u. & 0.0197748 & 0.0206528 & 0.0201948 & 0.018613 & 0.0166502 \\
\hline
\end{tabular}

The direct comparison of ab initio and classical free energy sampling results (see below for classical metadynamics) is problematic since we use essentially different collective variables (coordination umber of a cation at fixed distances for $a b$ initio and the cation - electrode plane distance for classical MD free energy sampling). However, we can directly compare the classical and $a b$ initio results when we compare the 1D free energy profiles resolved with respect to the $\mathrm{Mg}$ cation coordination number. In our previous work [17] we compared the coordination profiles (see Fig. 2 and comments to it) 
We next explore the state of charge of the $\mathrm{Mg}$ cation as it approaches the interface. For this purpose, we calculated the Bader charges of the $\mathbf{M g}$ cation. The results are shown in Fig. S3.

Figure S3. Averaged energies and Bader charges of the $\mathrm{Mg}$ cation for each distance calculated by averaging the energy over the AIMD trajectory for the global minima with respect to the cation coordination.

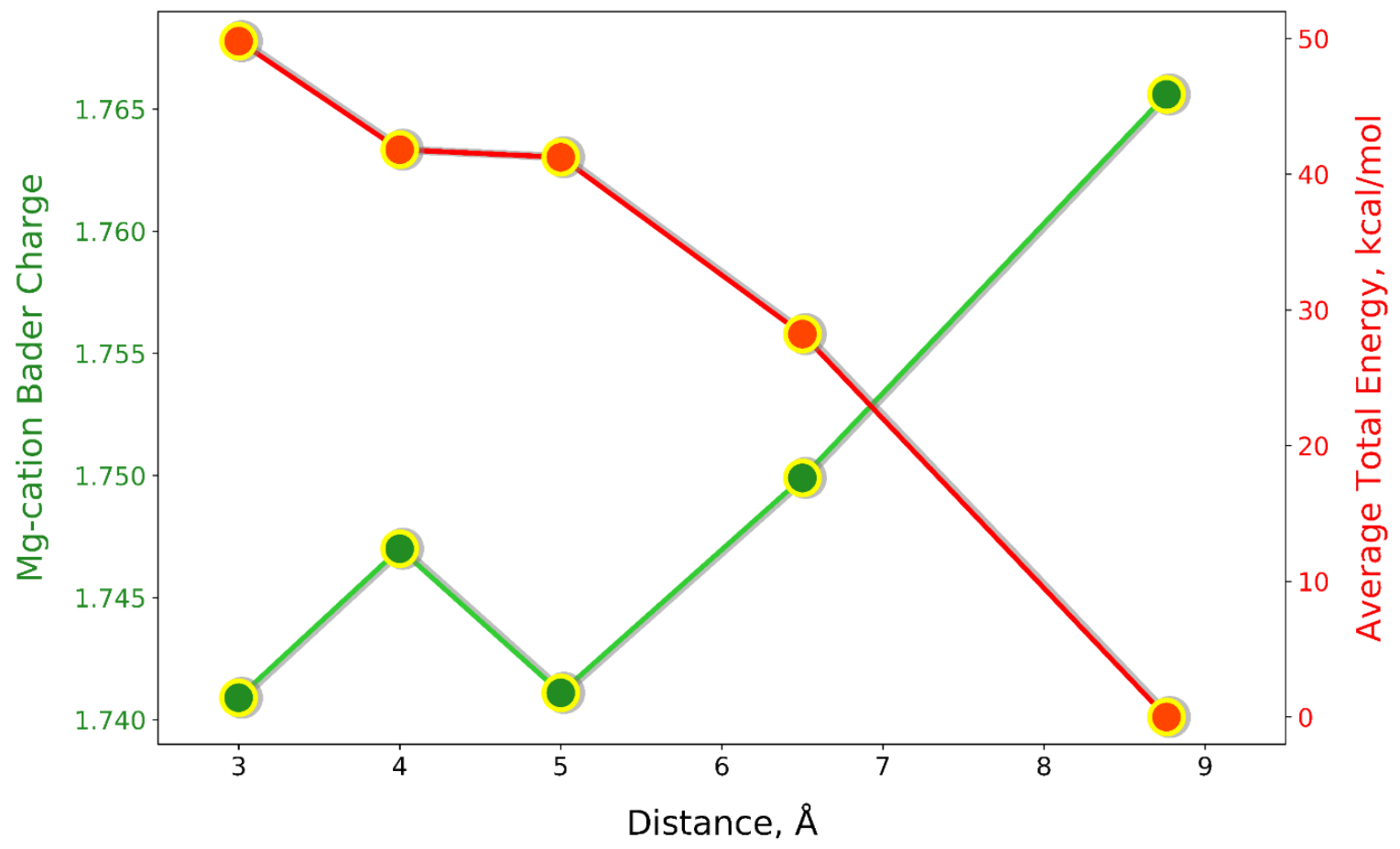

\section{Classical MD metadynamics protocol}

Equilibrated boxes of solvent molecules with ions (see regular classical MD protocol section) next to the neutral (negatively charged) graphene were used as initial configuration for the free energy sampling with the metadynamics protocol. We use various sets of collective variables (CV) to explore the free energy landscape of the free Mg-cation solvation in THF solvent: 1) the $\mathrm{Mg}$ cation coordination number, $\mathrm{CN}$, with respect to the oxygen atoms of THF solvent molecules; 2) the $\mathrm{Mg}$ cation coordination number, $\mathrm{CN}$, with respect to the oxygen atoms of TFSI anions; 3 ) the distance between the $\mathrm{Mg}$ cation and the plane of the graphene electrode; 4) the distance between the $\mathrm{Mg}$ cation and the center of mass of the $\mathrm{S}-\mathrm{N}-\mathrm{S}$ fragment of the TFSI anion. We used these CVs to construct 1D and 2D free energy profiles for isolated ions $\left(\mathrm{Mg}^{2+}, \mathrm{TFSI}^{-}\right)$, ion pairs $\left(\mathrm{MgTFSI}^{+}\right)$and other aggregates $\left(\mathrm{Mg}_{\mathrm{x}} \mathrm{TFSI}_{\mathrm{y}}\right)$.

In Tables S4-S6 we summarize the parameters of the metadynamics free energy sampling for various simulations: height of the Gaussian hills $(\mathrm{H}, \mathrm{kcal} / \mathrm{mol})$, frequency of hill creation (freq, steps), and width of hills (W, either $\AA$ for $\mathrm{CV}$ - distance or unitless for CV - coordination number) along with the ranges for the collective variables, the cut-off radius ( $\mathrm{r}_{\mathrm{o}}, \AA$ ) for $\mathrm{CN}$ and the overall simulation times (Time, nanoseconds) for each system. 
Table S4. Parameters of classical MD metadynamics free energy sampling.

\begin{tabular}{|c|c|c|c|c|}
\hline & $\begin{array}{l}\text { THF } \\
\text { Graphene-Distance } \\
\text { Fig. } 1 \\
\mathrm{~T}=298 \mathrm{~K} \\
\mathrm{~d}[1.5 ; 20] \AA \\
\end{array}$ & $\begin{array}{l}\mathrm{Mg}^{2+} / \mathrm{THF} \\
\text { Graphene-Distance } \\
\text { Fig. } 2 \\
\mathrm{~T}=298 \mathrm{~K} \\
\mathrm{~d}[1.5 ; 20] \AA \\
\end{array}$ & $\begin{array}{l}\text { TFSI-/THF } \\
\text { Graphene-Distance } \\
\text { Fig. } 2 \\
\text { T }=298 \mathrm{~K} \\
\mathrm{~d}[1.5 ; 20] \AA \\
\end{array}$ & $\begin{array}{l}\text { MgTFSI }^{+} / \mathrm{THF} \\
\text { Graphene-Distance } \\
\text { Fig. } 2 \\
\mathrm{~T}=298 \mathrm{~K} \\
\mathrm{~d}[1.5 ; 20] \AA \\
\end{array}$ \\
\hline $\mathrm{H}$ & 0.01 & 0.01 & 0.01 & 0.01 \\
\hline $\mathrm{W}$ & 0.05 & 0.05 & 0.01 & 0.01 \\
\hline freq & 1000 & 1000 & 500 & 1000 \\
\hline Time & 1000 & 1000 & 1200 & 1000 \\
\hline
\end{tabular}

Table S5. Parameters of classical MD metadynamics free energy sampling.

\begin{tabular}{|c|c|c|c|}
\hline & $\begin{array}{l}\mathrm{Mg}^{2+} / \mathrm{THF} \\
\text { CN-Graphene Distance } \\
\text { Fig. } 4 \\
\mathrm{~T}=298 \mathrm{~K} \\
\mathrm{~d}[1.5 ; 20] \AA \\
\mathrm{CN}[4.5 ; 6.5] \mathrm{r}_{\mathrm{o}}=3.07 \AA\end{array}$ & $\begin{array}{l}\text { MgTFSI }{ }^{+} / \text {THF/TFSI } \\
\text { CN-Graphene Distance } \\
\text { Fig. } 5 \\
\mathrm{~T}=298 \mathrm{~K} \\
\mathrm{CN} 1[0.0 ; 6.3], \mathrm{CN} 2[0.0 ; 4.0], \\
\mathrm{r}_{\mathrm{o} 1}=3.07 \AA, \mathrm{r}_{\mathrm{o}}=3.07 \AA ; \mathrm{d}[1.5 ; 12] \AA\end{array}$ & $\begin{array}{l}\text { MgTFSI } 2 \text { /finite concentration/THF } \\
\text { CN-Graphene Distance } \\
\text { Fig. } 6 \\
\mathrm{~T}=298 \mathrm{~K} \\
\mathrm{CN}[0.0 ; 6.3] ; \mathrm{r}_{\mathrm{o}}=3.07 \AA \\
\mathrm{d}[1.5 ; 10] \AA\end{array}$ \\
\hline $\mathrm{H}$ & 0.02 & 0.02 & 0.02 \\
\hline $\bar{W}$ & 0.05 & 0.05 & 0.05 \\
\hline freq & 200 & 200 & 200 \\
\hline Time & 900 & 800 & 900 \\
\hline
\end{tabular}

Table S6. Parameters of classical MD metadynamics free energy sampling.

\begin{tabular}{|c|c|c|c|c|c|}
\hline & $\begin{array}{l}\mathrm{Mg}^{2+} / \mathrm{THF} \\
\text { Negative } \\
\text { Graphene- } \\
\text { Distance } \\
\text { Fig. } 2 \\
\mathrm{~T}=298 \mathrm{~K} \\
\text { d }[1.5 ; 20] \AA\end{array}$ & $\begin{array}{l}\text { MgTFSI }^{+} / \text {THF } \\
\text { Negative } \\
\text { Graphene- } \\
\text { Distance } \\
\text { Fig. } 2 \\
\mathrm{~T}=298 \mathrm{~K} \\
\mathrm{~d}[1.5 ; 20] \AA\end{array}$ & $\begin{array}{l}\text { Mg1.6+/THF } \\
\text { CN-Graphene Distance } \\
\text { Fig. S5 } \\
\mathrm{T}=298 \mathrm{~K} \\
\mathrm{~d}[1.5 ; 20] \AA \\
\mathrm{CN}[4.5 ; 6.2] \quad \mathrm{r}_{\mathrm{o}}=3.07 \AA\end{array}$ & $\begin{array}{l}\text { MgTFSI }^{+} / \text {THF } \\
\text { CN-Graphene Distance } \\
\text { Fig. S6 } \\
\mathrm{T}=298 \mathrm{~K} \\
\mathrm{CN}[2.5 ; 6.3], \mathrm{r}_{\mathrm{o}}=3.07 \AA \\
\mathrm{d}[1.5 ; 20] \AA\end{array}$ & $\begin{array}{l}\text { MgTFSI }^{+} / \text {THF } \\
\text { Dissociation-Graphene } \\
\text { Distance } \\
\text { Fig. S6 } \\
\mathrm{T}=298 \mathrm{~K} \\
\mathrm{~d} 1[1.0 ; 12] \AA \\
\mathrm{d} 2[1.5 ; 12] \AA\end{array}$ \\
\hline $\mathrm{H}$ & 0.01 & 0.02 & 0.02 & 0.02 & 0.02 \\
\hline $\mathrm{W}$ & 0.05 & 0.05 & 0.05 & 0.05 & 0.05 \\
\hline freq & 500 & 200 & 200 & 200 & 200 \\
\hline Time & 2500 & 2500 & 800 & 1000 & 850 \\
\hline
\end{tabular}

As suggested [2], the convergence of the free energy sampling by the metadynamics protocol is reached when one reaches diffusive motion along the $\mathrm{CV}$. Here we used parameters of sampling ( $\mathrm{H}, \mathrm{W}$, freq and time) that guarantee [17] the convergence (diffusive regime) at the end of sampling time.

To distinguish the solvation population in the bulk solution from that at the interface, we introduce the $1 \mathrm{D}$ solvation profile of the $\mathrm{Mg}$ cation versus the $\mathrm{Mg}$ coordination number (with respect to the oxygens of THF molecules) averaged over the interfacial region $(4-6 \AA)$. Therefore, $\langle\Delta G(C N)\rangle_{d}=\frac{1}{d_{2}-d_{1}} \int_{d_{1}}^{d_{2}} \Delta G(C N, h) d h$, where $\mathrm{d}_{1}=4 \AA$, and $\mathrm{d}_{2}=6 \AA$, and bring the minimum of $\langle\Delta G(C N)\rangle_{d}$ to zero to be able to compare the relative population of species. The solvation profile in the bulk was set $\Delta G(C N)_{b u l k}=\Delta G(C N, h=10 \AA)$. Since the correct structure of the electric double layer is usually difficult to obtain for charged surfaces, we use $\Delta G(C N, h=10 \AA)$ calculated for a finite concentration $(0.12 \mathrm{M}-0.25 \mathrm{M})$ for neutral graphene as $\Delta G(C N)_{\text {bulk }}$ (see black dashed, red and blue curves in Figure 6, main text). We do not expect that the range of solvation structures of the $\mathrm{Mg}$ cation would change with further increase of the salt concentration unless we reach the very high (beyond the solubility limit) concentration. 


\section{Polarization effect on the graphene induced by the Mg-cation in THF}

We used the ab initio constrained MD (umbrella sampling) trajectory to evaluate the effect of polarization induced by the $\mathrm{Mg}$ cation on the charge distribution on the graphene sheet. Specifically, we used a snapshot for AIMD with the Mg cation at $h=3 \AA$ from the graphene plane. Then we calculate the Bader charges for $\mathrm{C}$-atoms of graphene. The results are shown in Figure S4.

Figure S4. The effect of polarization of graphene by the $\mathrm{Mg}$ cation. The graphene polarization is expected to be maximal for the least screened Mg cation (3-fold coordination state at $h=3 \AA$ ). The charges of $\mathrm{C}$-atoms are coded by color scaled between -0.15 to +0.15 e. a) The differential charge distribution (in units of e) for $\rho_{i} \leq \rho \leq \rho_{i+1}$, where $\rho$-is a distance in the graphene plane from the normal that connects the $\mathrm{Mg}$ cation and the plane, and $\rho_{i}, \rho_{i+1}-$ are the internal and external radii of a ring ( $1 \AA \AA$ width). b) The integral charge as a function of distance in the graphene plane from the normal that connects the $\mathrm{Mg}$ cation and the plane. c) Charge distribution for bare Mg-cation above the graphene.

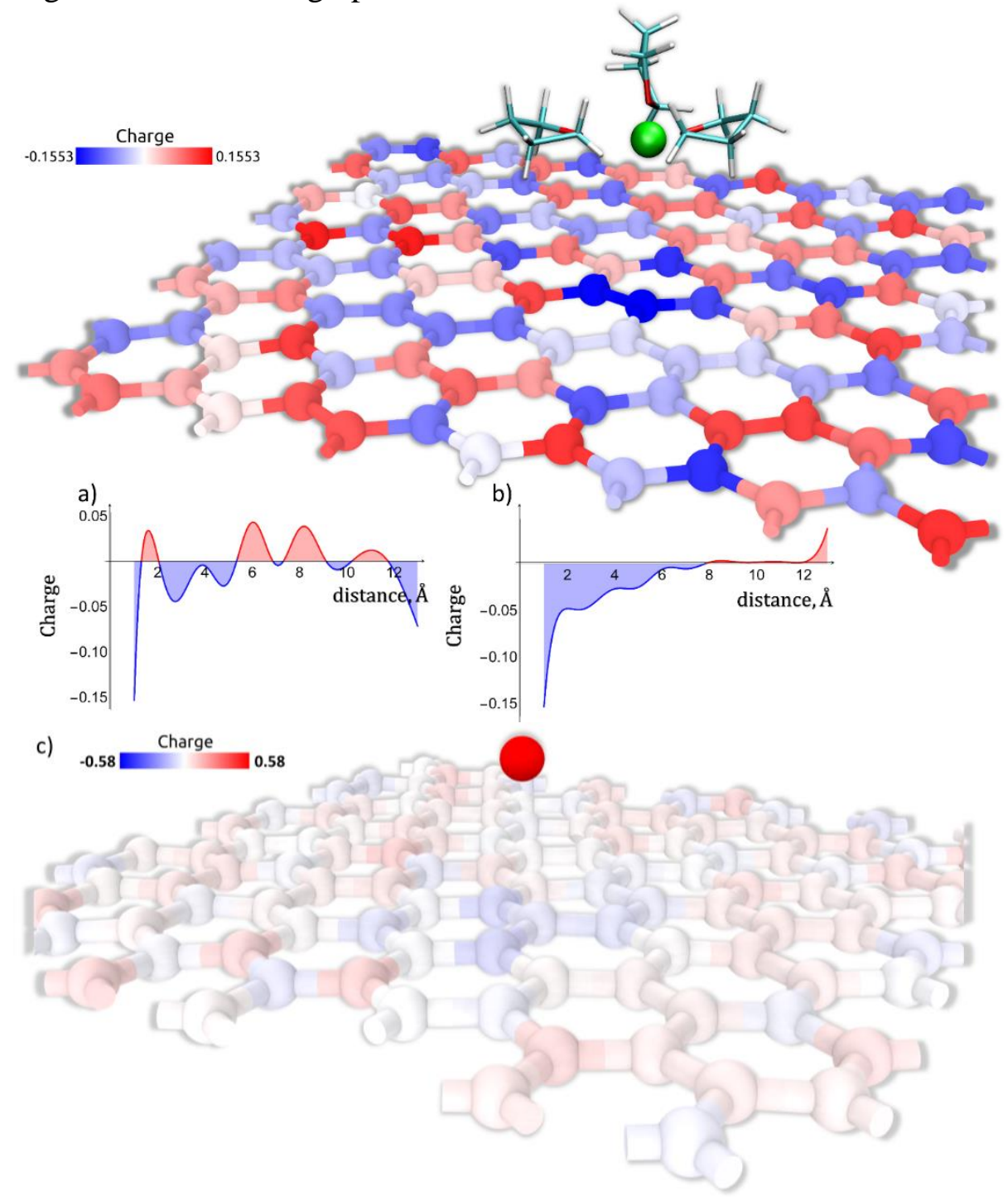


The effect of the rescaling the $\mathrm{Mg}$ cation charge from $2+$ to $1.6+$ is shown in Figure $\mathbf{S 5}$.

Figure S5. 2D free energy surfaces resolved with respect to the coordination number and the distance to the graphene electrode plane for the free $\mathrm{Mg}^{1.6+}$ cation in THF at $\mathrm{T}=298 \mathrm{~K}$ next to the neutral graphene. The coordination number accounts the number of THF oxygen atoms in the first solvation sphere. The labeled most stable 5- and 6-fold solvation configurations in the vicinity to the neutral graphene are shown. (compare with Figure 4, main text).

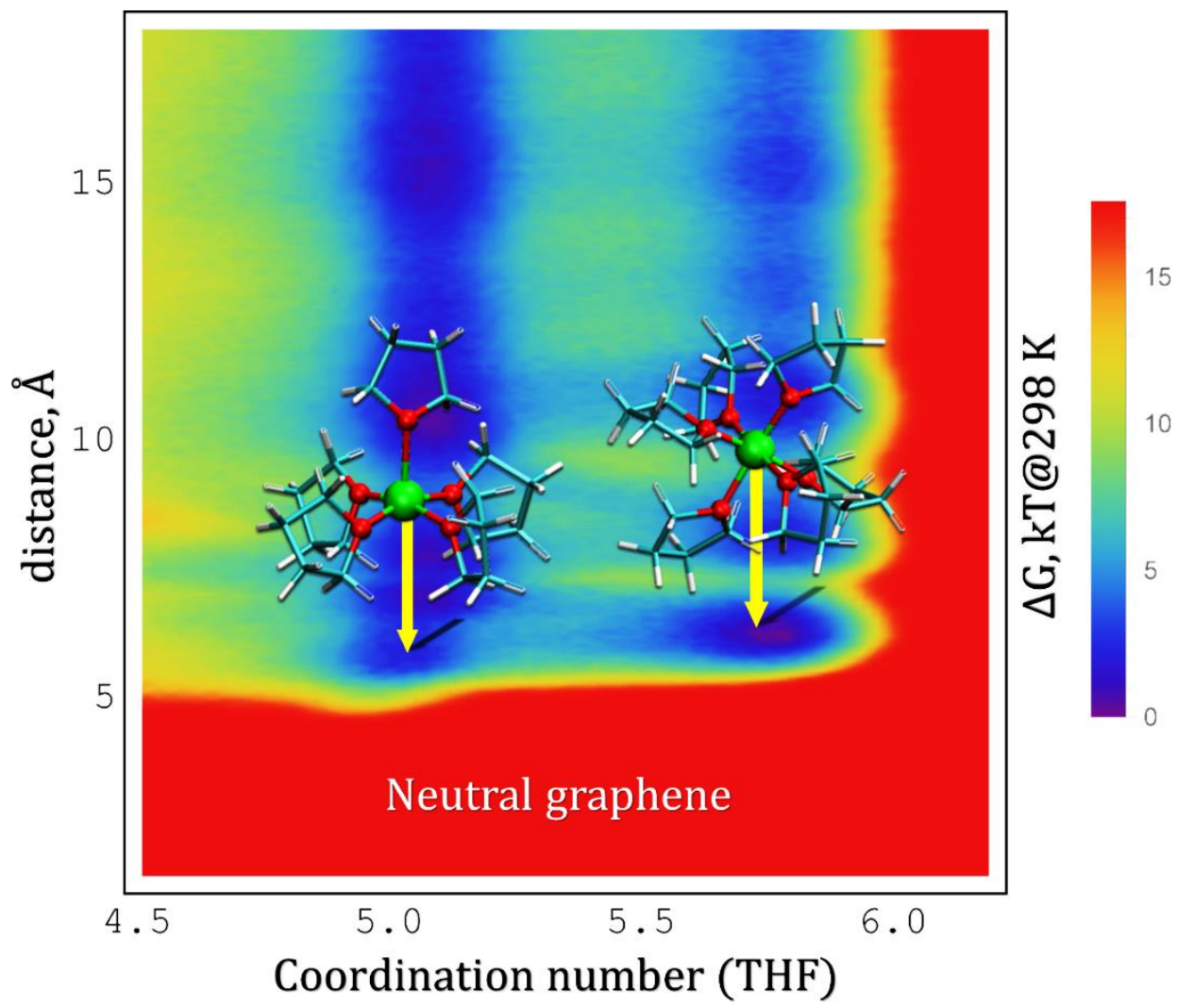

\section{Solvation structure dynamics of the MgTFSI $^{+}$ion pair in THF next to the negatively charged graphene}

We use classical MD based metadynamics free energy sampling and analyze the solvation structure of the isolated MgTFSI ${ }^{+}$ion pair in THF next to negatively charged graphene (compare Figure 5, main text) from two perspectives: 1) what solvation configuration is the most efficient in delivering the $\mathrm{Mg}$ cation to the interface, and 2) what does the dissociation of the ion pair next to the electrode looks like? To this ends, we resolve the 2D free energy surface as a function of the cation distance to the graphene plane and its coordination with THF (see left panel Figure S6) and the cation - anion distance (see right panel Figure S6). 
Figure S6. 2D free energy surfaces resolved with respect to the coordination number of oxygens of THF molecules and the distance to the plane of the negatively charged graphene electrode (left panel) and the cation - TFSI anion distance and the distance to the plane of the negatively charged graphene electrode (right panel) for the isolated $\mathrm{MgTFSI}^{+}$ion-pair in THF at $\mathrm{T}=298 \mathrm{~K}$.
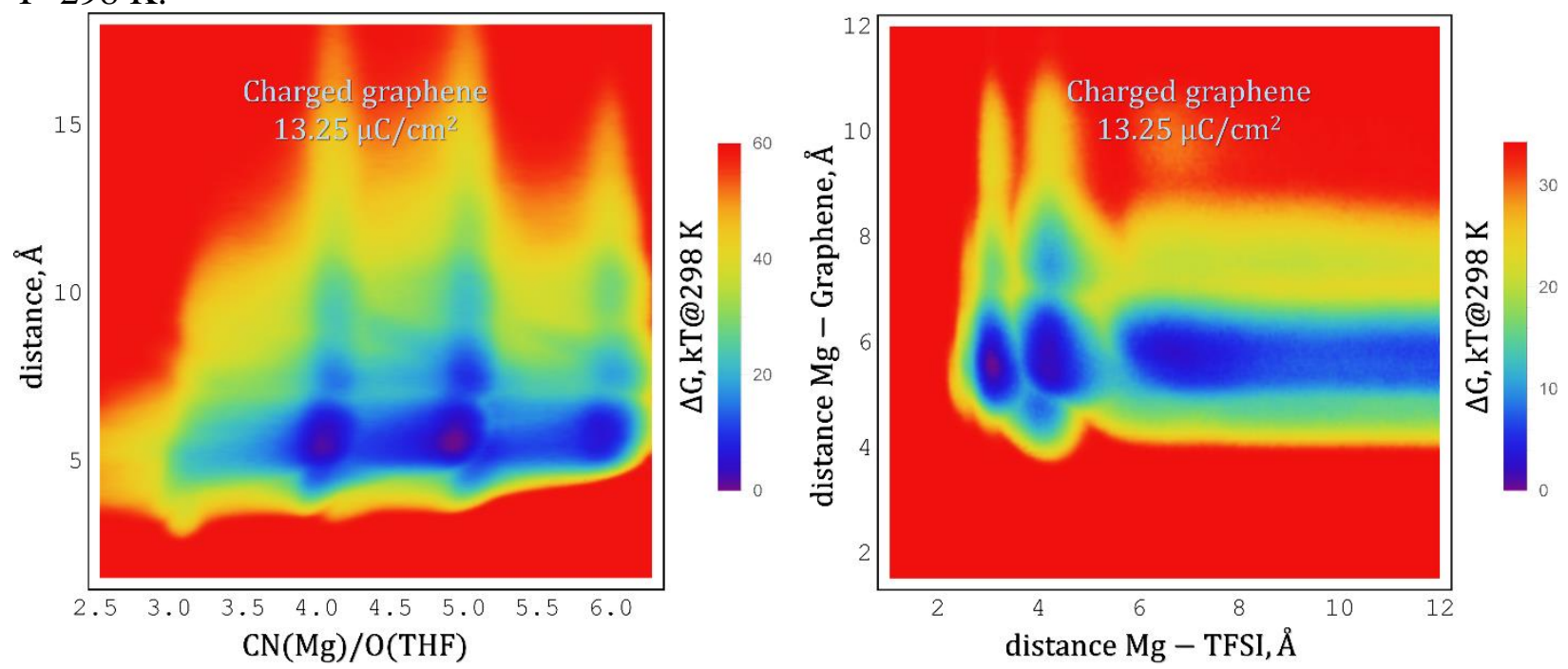

\section{Force Fields and Charges}

Standard Charges for Elements in the simulations:

TFSI-anion

N -0.58800

S 0.91100

O -0.46100

C 0.31900

F $\quad-0.13800$

THF

C1 0.12740

C2 -0.10640

O -0.42760

H1 0.05170

H2 0.04470

G2

O1 -0.42560

O2 -0.42460

C1 0.11870

C2 0.12440

C3 0.13040

H1 0.03370

$\mathrm{H} 2 \quad 0.03970$

H3 0.04170 


\section{Force Fields}

\section{Mg-cation/THF}

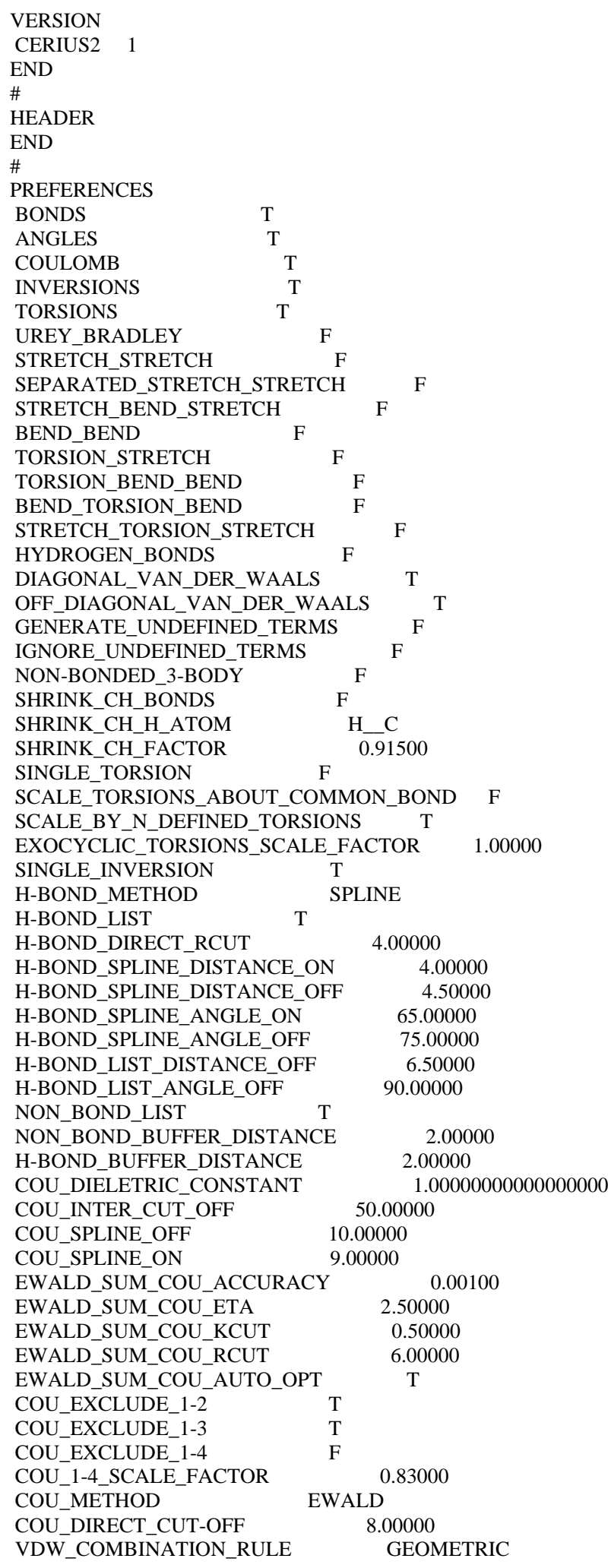




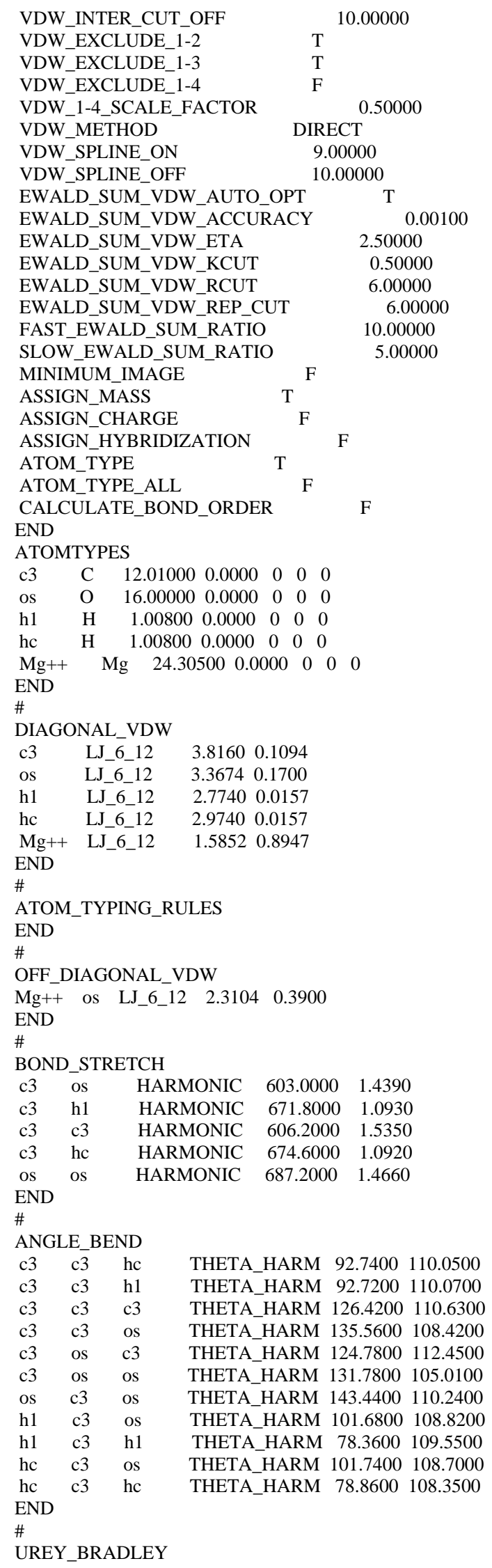




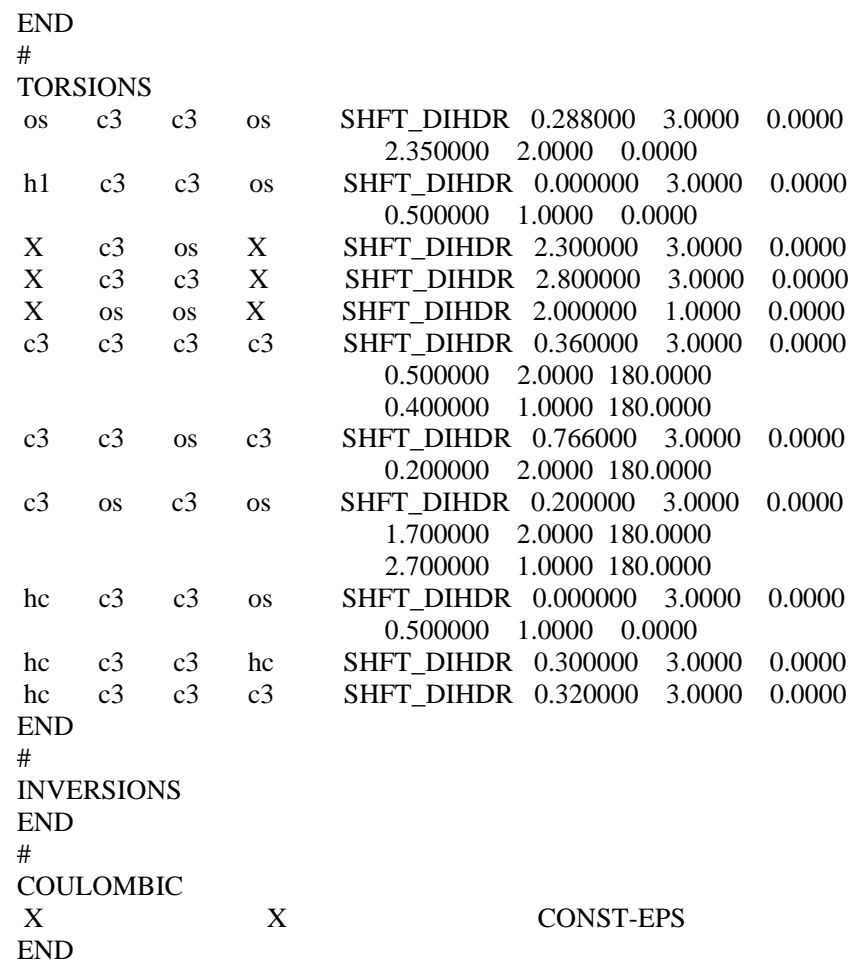

\section{TFSI-anion}

VERSION

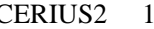




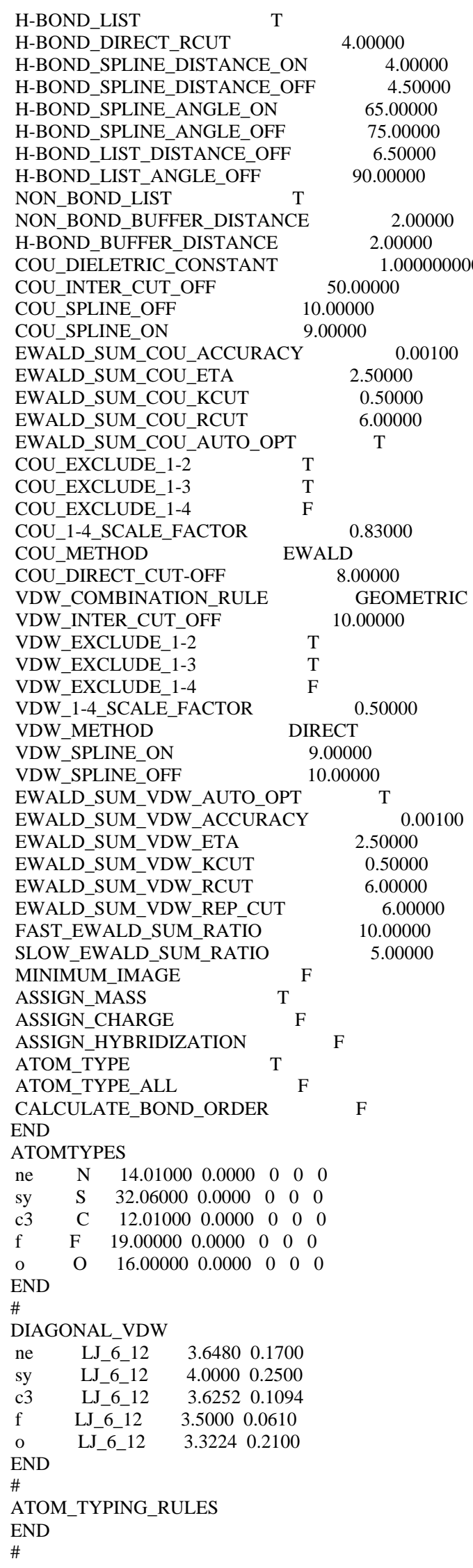




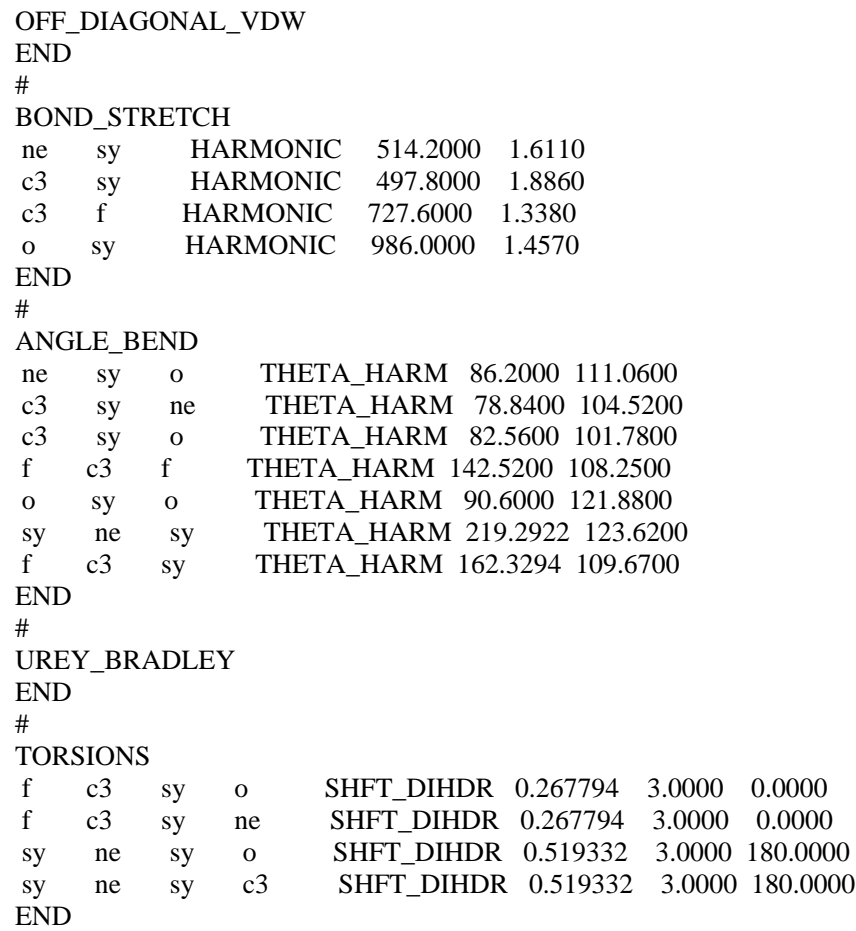

\section{Graphene \\ VERSION \\ CERIUS2 1 \\ END \\ \# \\ HEADER}

The field is a combination of the origin graphite force field with Naoki's PVD, Mario's Teflon and tap's F3C water force fields (optimized to have correct $\mathrm{HOH}$ and $\mathrm{OH}$ stretches and hvap) for simulating graphene and congugated graphene $(\mathrm{H}, \mathrm{F}, \mathrm{OH})$ systems. 


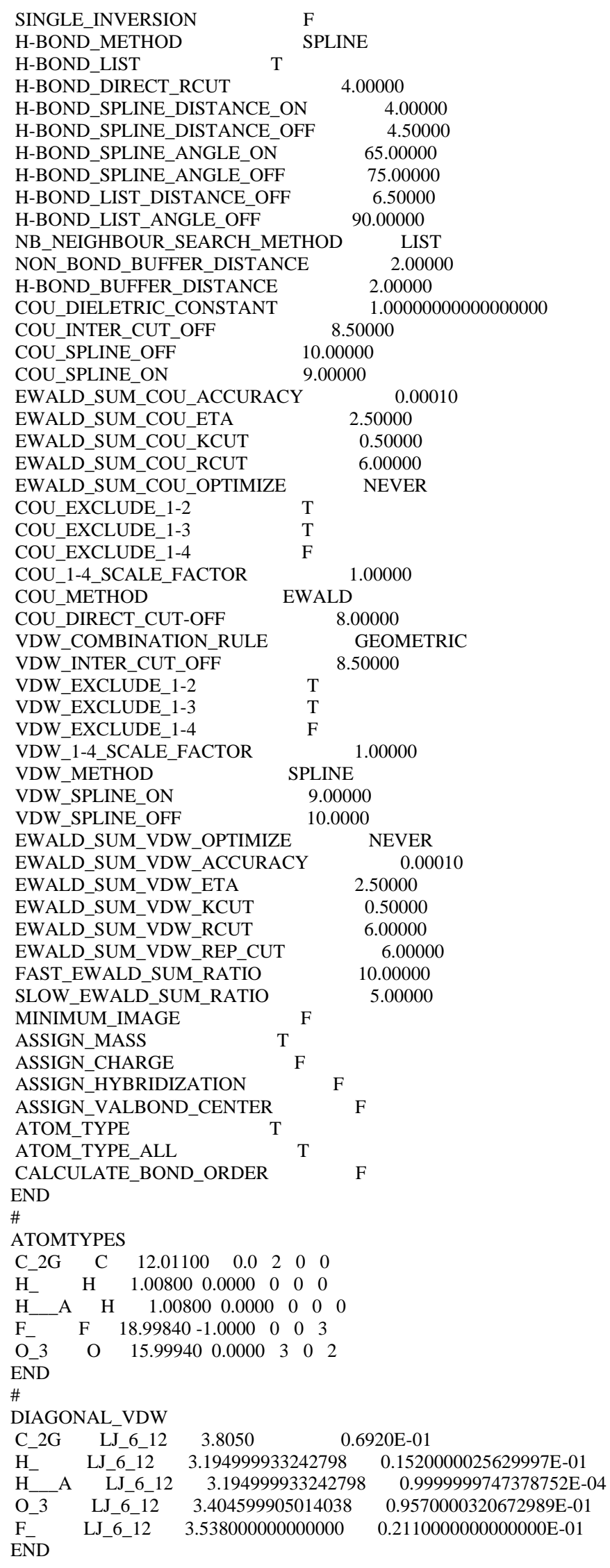




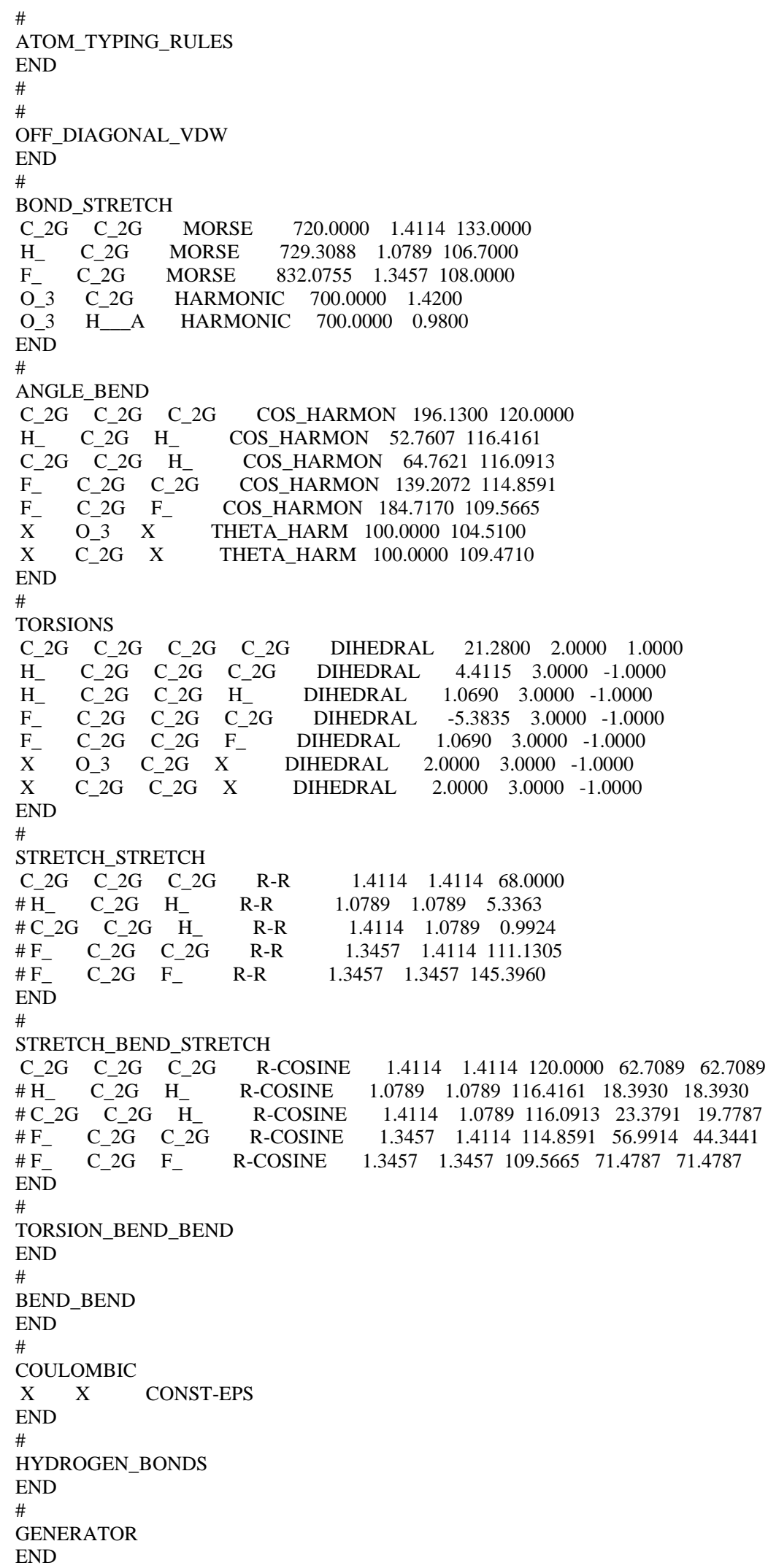




\section{References}

[1] A. Laio, F. L. Gervasio, Rep. Prog. Phys. 2008, 71, 126601.

[2] A. Barducci, M. Bonomi, M. Parrinello, Wiley Inter. Rev.: Comp. Mol. Sci. 2011, 1, 826-843.

[3] J. Kästner, Wiley Inter. Rev.: Comp. Mol. Sci. 2011, 1, 932-942.

[4] A. L. Ferguson, J. Compt. Chem. 2017, 38, 1583-1605.

[5] J. Wang, R. M. Wolf, J. W. Caldwell, P. A. Kollman, D. A. Case, J. Comput. Chem. 2004, 25, 1157.

[6] J. Aqvist, J. Phys. Chem. 1990, 94, 8021-8024.

[7] N. Karasawa, S. Dasgupta and W. A. Goddard III, J. Phys. Chem. 1991, 95, 6, 2260-2272.

[8] S. Plimpton, J. Comp. Phys. 1995, 117, 1.

[9] S. A. Nosé, J. Chem. Phys. 1984, 81, 511.

[10] W. G. Hoover, Phys. Rev. A: At. Mol. Opt. Phys. 1985, 31, 1965.

[11] G. J. Martyna, M. L. Klein, M. Tuckerman, J. Chem. Phys. 1992, 97 (4), 2635-2643.

[12] J. Hutter, M. Iannuzzi, F. Schiffmann, J. VandeVondele, Wiley Interdiscip. Rev. Comput.

Mol. Sci. 2014, 4, 15-25.

[13] J. VandeVondele, J. Hutter, J. Chem. Phys., 2007, 127, 114105.

[14] S. Goedecker, M. Teter, J. Hutter, Phys. Rev. B 1996, 54(3), 1703-1710.

[15] S. Grimme, J. Antony, S. Ehrlich, H. Krieg, J. Chem. Phys. 2010, 132(15), 154104.

[16] D. Trzesniak, A.-P. E. Kunz, W.F. van Gunsteren, ChemPhysChem, 2007, 8, 162-169.

[17] A. Baskin and D. Prendergast, J. Phys. Chem. Lett. 2020, 11, 9336-9343. 